\title{
Waveguide arrays and spectral filtering for multi-frequency mode- locked pulse sources
}

Edward Farnum, Brandon G. Bale, J. Nathan Kutz

Edward Farnum, Brandon G. Bale, J. Nathan Kutz, "Waveguide arrays and spectral filtering for multi-frequency mode-locked pulse sources," Proc. SPIE 7386, Photonics North 2009, 73862E (4 August 2009); doi:

$10.1117 / 12.837773$

SPIE. Event: Photonics North 2009, 2009, Quebec, Canada 


\title{
Waveguide Arrays and Spectral Filtering for Multi-Frequency Mode-locked Pulse Sources
}

\author{
Edward Farnum ${ }^{a}$, Brandon G. Bale ${ }^{b}$ and J. Nathan $\mathrm{Kutz}^{c}$ \\ ${ }^{a}$ Center for Science, Technology, and Mathematics, Kean University, Union, NJ 07083; \\ ${ }^{b}$ Photonics Research Group, Aston University, Birmingham UK B4 7ET; \\ ${ }^{c}$ Department of Applied Mathematics, University of Washington, Seattle, WA 98195-2420
}

\begin{abstract}
Current optical fiber-communication networks increasingly rely on wavelength-division multiplexing (WDM) technologies in conjunction with optical time-division multiplexing (OTDM) of individual WDM channels. The combination of high-repetition-rate data streams with a large number of WDM channels has pushed transmission rates to nearly $1 \mathrm{~TB} / \mathrm{s}$, creating a demand for all-optical transmission sources that can generate pico-second modelocked pulses at various wavelengths. Through nonlinear mode-coupling in a wave-guide array and a periodically applied multi-notch frequency filter, robust multi-frequency mode-locking can be achieved in a laser cavity in both the normal and anomalous dispersion regimes. We develop a theoretical description of this multiplewavelength mode-locking, and characterize the mode-locked solutions and their stability for an arbitrary number of frequency channels. The theoretical investigations demonstrate that the stability of the mode-locked pulse solutions of multiple frequency channels depends on the degree of inhomogenity in gain saturation. Specifically, only a small amount of inhomogeneous gain-broadening is needed for multi-frequency operation in the laser. In this presentation, the conditions on the system parameters necessary for generating stable mode-locking is explored for arbitrary number of frequency channels. The model suggests a promising source for multi-frequency photonic applications.
\end{abstract}

Keywords: Mode-locked lasers, wavelength-division multiplexing, waveguide arrays

\section{INTRODUCTION}

Current optical fiber-communication networks increasingly rely on wavelength-division multiplexing (WDM) technologies in conjunction with optical time-division multiplexing (OTDM) of individual WDM channels. The combination of high-repetition-rate data streams with a large number of WDM channels has pushed transmission rates to nearly $1 \mathrm{~TB} / \mathrm{s}^{1}$ This has created a demand for all-optical transmission sources that can generate picosecond mode-locked pulses at various wavelengths. ${ }^{2}$ We develop a theoretical description, based upon wave-guide array technology, of this multiple-wavelength mode-locking, and characterize the mode-locked solutions and their stability for an arbitrary number of frequency channels. Specifically, through nonlinear mode-coupling in a waveguide array and a periodically applied frequency filter, robust multi-frequency mode-locking can be achieved in a laser cavity.

For pulsed lasers, the past decade has focused on the use of Erbium-doped fibers in actively and passively mode-locked fiber lasers operating in both the normal and anomalous dispersion regimes (see [3] and references therein). This is in part due to the minimal loss of optical fibers at $1550 \mathrm{~nm}$, and the maturation of Erbium-doped fiber as the basic component for optical lasers and amplifiers. Many passive modelocking schemes have been successfully demonstrated including modelocking via nonlinear interferometry in the figure eight laser, ${ }^{4-7}$ polarization rotation in the ring laser, ${ }^{8-11}$ and a linear cavity configuration which utilizes the fast saturable absorption of a semiconductor structure. ${ }^{12-15}$ The key underlying feature of each modelocked laser is the intensity-discrimination which is achieved by the modelocking mechanism. ${ }^{16}$ The additional loss imparted upon low-intensity portions of the pulse, whether it be dispersive radiation or the wings of a pulse, gives the

Further author information: (Send correspondence to J.N.K.)

J.N.K.: E-mail: kutz@amath.washington.edu, Telephone: 12066853029

Photonics North 2009, edited by Réal Vallée, Proc. of SPIE Vol. 7386, 73862E (C) 2009 SPIE · CCC code: 0277-786X/09/\$18 · doi: 10.1117/12.837773 
necessary pulse shaping required to achieve stable modelocking operation. ${ }^{16}$ This loss of the electromagnetic field is balanced with the Erbium amplifier, thus allowing for stable and robust mode-locking.

The intensity-discrimination considered here results from the nonlinear mode-coupling in a wave-guide array. ${ }^{17,18}$ The mode-locking operation for this wave-guide array based laser generates robust and stable modelocked pulses which are global attractors to the cavity in both the normal and anomalous dispersion regimes. To generate multi-frequency mode-locking, we propose to place a multi-notched spectral filter in the laser cavity. This results in a periodic, frequency-dependent attenuation of the electromagnetic field. This frequency selection mechanism allows for multi-frequency mode-locking.

\section{GOVERNING EQUATIONS}

The evolution of the electromagnetic field in the laser cavity is subject to several key physical effects. In addition to the inherent effects of chromatic dispersion and self-phase modulation, the laser cavity provides a saturating gain to counteract the net laser cavity losses. Furthermore, successful mode-locking requires some form of intensity discrimination (saturable absorption) to generate ultra-short pulses. We present the governing equations in the laser cavity which includes a wave-guide array for the intensity discrimination. ${ }^{17,18}$ In order to characterize the stability of the mode-locked solutions, we consider a frequency-component reduction of the governing equations which allow for the construction and characterization of exact mode-locked solutions. This gives a comprehensive theory and treatment of the multi-frequency mode-locking behavior.

The inclusion of chromatic dispersion, self-phase modulation, attenuation, bandwidth limited gain, and an intensity-discrimination element comprise the key components of a laser cavity. Averaging over all these physical effects that occur per round trip in the laser cavity, a master mode-locking model has been developed that uses generic nonlinear loss to provide the necessary intensity discrimination. ${ }^{16}$ Using a more physically realistic mechanism for intensity discrimination, an equivalent mode-locking model has been developed based upon the nonlinear mode-coupling (NLMC) in a wave-guide array.${ }^{17-19}$ The governing equations can be shown, via an averaging method, to result in a coupled set of partial differential equations for the electric field in each of the coupled wave-guides. ${ }^{19}$ Unlike the master-modelocking model, however, this model is quantitatively accurate.

Figure 1 shows a typical ring cavity configuration which includes these physical effects in addition to a spectral filter for achieving multi-frequency operation (Fig. 1b). It is important to note that in this Subsection, the spectral-filtering is not included in the average equations. Rather, the spectral filtering is applied once every round trip of the laser cavity. The coupled wave-guide fields are governed by ${ }^{20}$

$$
\begin{aligned}
& i \frac{\partial u}{\partial Z}+\frac{D}{2} \frac{\partial^{2} u}{\partial T^{2}}+\beta_{0}|u|^{2} u+C v \\
&+i \gamma_{0} u-i g(Z)\left(1+\tau \frac{\partial^{2}}{\partial T^{2}}\right) u=0 \\
& i \frac{\partial v}{\partial Z}+\frac{D}{2} \frac{\partial^{2} v}{\partial T^{2}}+\beta_{1}|v|^{2} v+C(u+w)+i \gamma_{1} v=0 \\
& i \frac{\partial w}{\partial Z}+\frac{D}{2} \frac{\partial^{2} w}{\partial T^{2}}+\beta_{2}|w|^{2} w+C v+i \gamma_{2} w=0
\end{aligned}
$$

with the bandwidth limited gain given by

$$
g(Z)=\frac{2 g_{0}}{1+\|u\|^{2}}
$$

Here $u, v$, and $w$ are the electric field envelopes in the center waveguide (zeroth) $(u)$, the first $(v)$ and second $(w)$ wave-guides respectively, ${ }^{19} Z$ is the propagation distance in the boosted frame of reference, $T$ is the time normalized by the characteristic full-width half-maximum mode-locked pulse width, and $\|u\|^{2}=\int_{-\infty}^{\infty}|u|^{2} d T$. The fields and space-time variables have been normalized via standard soliton scalings. ${ }^{17-19}$ The parameters $\beta_{i}$, $C, \gamma_{i}$, and $\tau$ correspond to the normalized values of the average self-phase modulation in the appropriate waveguide channel, average linear wave-guide coupling per round trip of the laser cavity, the loss in the appropriate wave-guide channel and the amplifying gain bandwith. In the soliton normalization, ${ }^{17-19}$ the parameter $D= \pm 1$ 


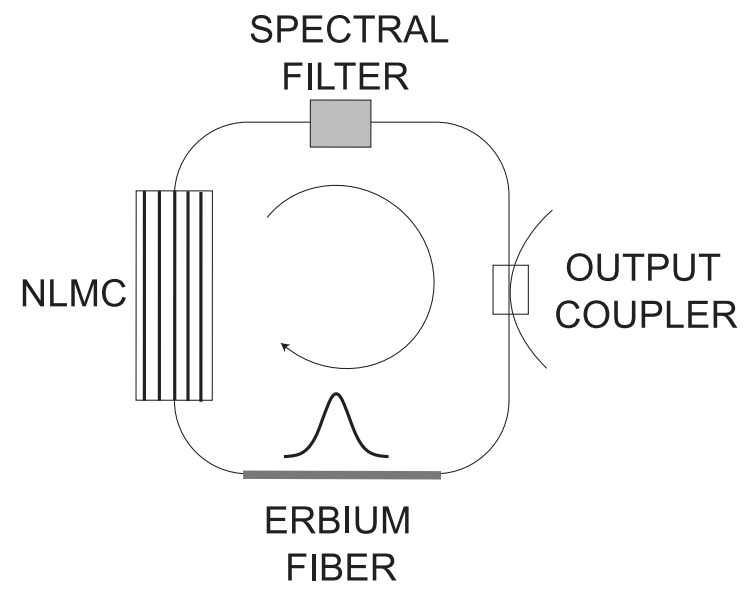

(a)

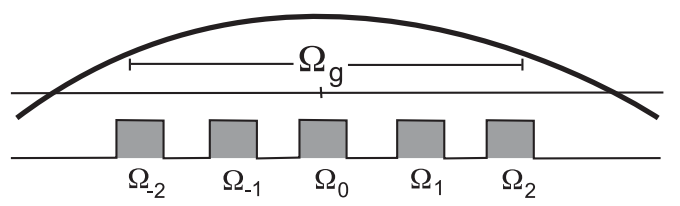

(b)

Figure 1. (a) Physical representation of the laser cavity which includes an erbium doped amplifier (bandwidth limited gain), output coupler, spectral filter, and wave-guide array. The wave-guide array provides the necessary intensity discrimination for mode-locking via nonlinear mode-coupling (NLMC). (b) Spectral representation of the gain-loss mechanisms in the laser cavity. Illustrated is a five notch-filter with individual notch widths $\Omega_{i}$, and the parabolic gain-bandwidth with FWHM $\Omega_{g}$. The filter allows transmission of certain spectral components, thus acting as a selection mechanism for propagation modes. This allows for robust and stable multi-frequency mode-locking.

determines wether we are in the anomalous $(D=1)$ or normal $(D=-1)$ group velocity dispersion $(\mathrm{GVD})$ regime. Note that the coupling between neighboring waveguides is due to the evanescent field. Provided the waveguides are sufficiently well-separated, the coupling is linear as proposed.

Equations (1) include all the dominant physical effects for single-frequency pulse generation. Proctor and $\mathrm{Kutz}^{19}$ showed that stable and robust mode-locking occurs in this model for physically realistic parameter values. ${ }^{19}$ Indeed, the wave-guide array as a mode-locking element provides a quantitative, asymptotically valid model which generates a mode-locked pulse solution as a global attractor. In multi-frequency mode-locking, the idea is to simultaneously generate mode-locked pulses at more than a single carrier frequency. Figure 1(b) shows the spectral filtering which is applied periodically to the laser cavity. For multi-frequency operation ( $N$-channels), it is imperative that the gain-bandwidth $\left(\Omega_{g}\right)$ be substantially larger than the spectral-filtering width $\Omega_{i}(i=1,2, \cdots, N)$ of the individual frequency channels. The notch-frequency filter acts as a selection mechanism which allows only a limited number of frequencies to persist in the laser cavity. With this simple filter design and its application once per round trip, robust and stable mode-locking can be achieved at all the frequencies allowed by the filter. Additionally, the configuration can be modified so as to favor only a limited number of frequencies.

To make further connection with experiment, recent work by Wise and co-workers ${ }^{21-23}$ has demonstrated robust mode-locking with intra-cavity filter insertion. Indeed, by increasing the gain in the cavity to overcome the additional attenuation due to the spectral filtering, they find excellent performance characteristics for their laser. In such a configuration, the filter notches are easily tunable in the range from 4-8 nanometers. For a typical erbium-doped fiber gain bandwidth of 30 nanometers, for instance, it is conjectured that placing a filter 


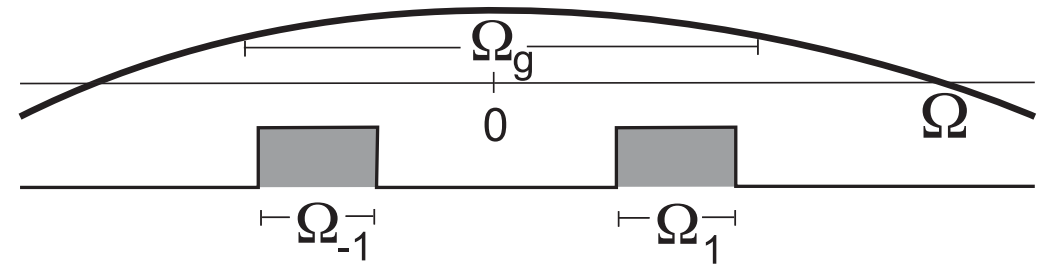

Figure 2. Spectral transmission profile of a dual notch-filter $\left(\Omega_{ \pm 1}\right)$ in comparision with the spectral gain bandwidth $\left(\Omega_{g}\right)$.

with two transmission windows of 4-8 nanometers spaced apart (center-to-center) by 10 nanometers would lead to dual-frequency mode-locked operation. For a larger number of channels, the filter windows must either be narrowed to fit more channels under the gain bandwidth, or gain-flattened erbium amplifier technologies must be used to broaden the gain bandwidth window. In theory, the spectral filtering must allow sufficient spacing between neighboring frequency channels in order to avoid cross-talk due to nonlinear frequency mixing and other pernicious effects. This imposes a limit on how tightly packed the various frequency channels can be before the multi-frequency mode-locking theory breaks down. In addition, the intra-cavity filtering must occur rapidly enough in order to avoid the significant spectral broadening that occurs due to self-phase modulation. This imposes a second fundamental constraint on the repetition rate of the laser cavity. Regardless, the spectral filtering technology is feasible and has been demonstrated for use in single-frequency operation. ${ }^{21-23}$

\section{MODE-LOCKING DYNAMICS}

Numerical simulations of Eq. (1) and (2) are performed using a spectral discretization in the time variable $T$ and a 4 th-order Runge-Kutta method for stepping forward in the propagation variable $Z$. The simulations explicitly demonstrate that the mode-locked solutions act as global attractors in the laser cavity. In what follows, special emphasis is given to the dual- and five-frequency mode-locking in both the normal and anomalous dispersion regimes. These two examples illustrate all the key features of the multi-frequency mode-locking dynamics. For the simulations that follow, we choose the physically relevant values $\beta_{0}=8, \beta_{1}=\beta_{2}=4, C=5, \gamma_{0}=\gamma_{1}=0$, and $\gamma_{2}=10 .{ }^{20}$ The values associated with the gain bandwidth and spectral filtering are given in the individual simulations below. It should be noted that during one cavity round-trip, the pulse undergoes large deformations due to the filtering, amplification, and pulse-shaping.

\subsection{Dual Filter Application}

The simplest realization of multi-frequency mode-locking is the case of two frequencies. Figure 2 illustrates the basic spectral interplay between the spectral filter $\left(\Omega_{-1}\right.$ and $\left.\Omega_{1}\right)$ and bandwidth-limited gain $\left(\Omega_{g}\right)$ which is determined by $\tau$. For the dual-frequency mode-locking illustrated here, individual notches are approximately one-fourth of the FWHM gain bandwidth, i.e. the filters would be 6-8 nanometers for a 24-32 nanometer gain bandwidth.

Figure 3 illustrates the mode-locking dynamics in the normal dispersion regime with $g_{0}=4.3$. The normalized gain bandwidth is characterized by $\tau=0.1$ so that the FWHM $\Omega_{g}=4.4$ (see Fig. 2). The filter widths are $\Omega_{ \pm 1}=1$ with a center position at $\Omega= \pm 1$ respectively. The spectral filter is applied every $Z=10$ units. The plot of the spectrum and time-evolution is shown one unit in $Z$ after filtering, i.e. this is equivalent to an output coupler. The figure clearly shows the evolution settles to the desired mode-locked solution. Recall that during one cavity round-trip, the pulse undergoes large deformations due to the filtering, amplification, and pulse-shaping. However, at the output coupler, the temporal evolution demonstrated in Fig. 3(b)-(c) are locked into a steady bound-state. The spectral evolution in Fig. 3(a) also clearly demonstrates the dual-frequency operation. The mode-locking in the normal regime is highly robust and produces stable mode-locking for a wide range of parameter space.

Figure 4 illustrates the mode-locking dynamics in the anomalous dispersion regime with $g_{0}=1.8$. The normalized gain bandwidth is characterized by $\tau=0.01$ so that the FWHM $\Omega_{g}=14.1$. The filter widths are 


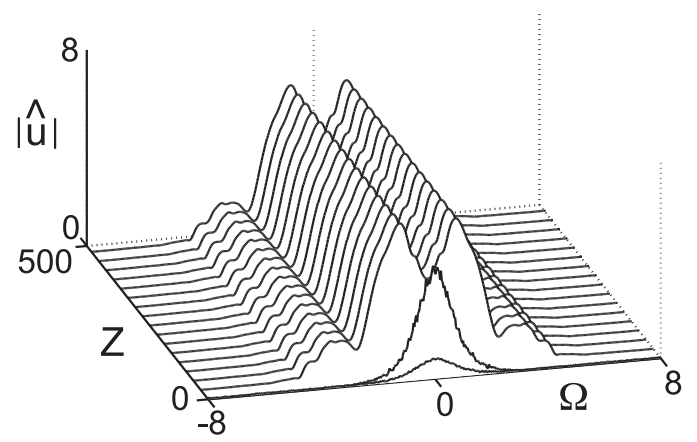

(a)

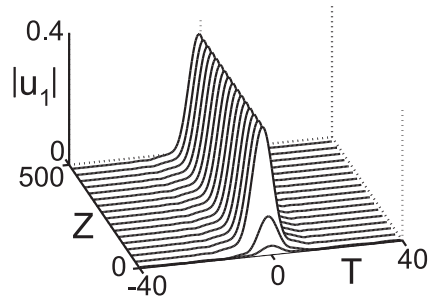

(b)

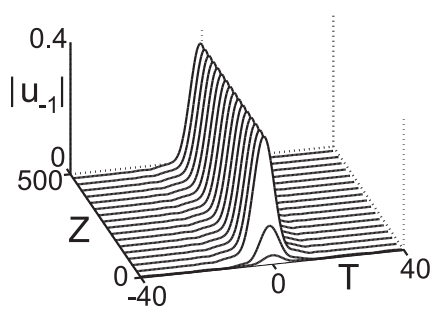

(c)

Figure 3. Illustration of the mode-locking dynamics in the normal dispersion regime with $g_{0}=4.3$. The normalized gain bandwidth is characterized by $\tau=0.1$ so that the FWHM $\Omega_{g}=4.4$. The filter widths are $\Omega_{ \pm 1}=1$ with a center position at $\Omega= \pm 1$ respectively. The spectral filter is applied every $Z=10$ units. The plot of the spectrum (a) and time-evolution (b)-(c) is shown one unit in $Z$ after filtering.

$\Omega_{ \pm 1}=4$ with a center position at $\Omega= \pm 4$ respectively. The spectral filter is applied every $Z=15$ units. The plot of the spectrum and time-evolution is shown 0.5 units in $Z$ after filtering. Again the evolution settles to the desired steady bound-state solution shown in Fig. 4(b)-(c). The spectral evolution in Fig. 3(a) again demonstrates the dual-frequency operation. The sharper separation in frequencies from the normal regime is largely due to the more immediate output coupling following the spectral filtering. In the anomalous regime, stable operation requires a small filter to output distance compared to the filter to filter period. Although the mode-locking in the anomalous regime starts from white-noise, its range of stable operation as a function of parameter space is much less robust. This suggests that special care must be taken in constructing an anomalous dispersion laser cavity with spectral filtering.

\subsection{Multi-Filter Application}

To explore beyond dual-frequency operation, we consider a five-channel mode-locking configuration. Figure 1(b) illustrates the spectral filtering components due to filter application $\left(\Omega_{j}\right.$ where $\left.j=-2,-1,0,1,2\right)$ and bandwidthlimited gain $\left(\Omega_{g}\right)$. For the 5-frequency mode-locking illustrated here, the individual notches are approximately one-sixth of the FWHM gain bandwidth, i.e. the filters would be 4-5.3 nanometers for a 24-32 nanometer gain bandwidth.

Figure 5 illustrates the mode-locking dynamics in the normal dispersion regime with $g_{0}=4.3$. The normalized gain bandwidth is characterized by $\tau=0.1$ so that the FWHM $\Omega_{g}=4.4$ (see Fig. 1(b)). The filter widths are $\Omega_{j}=0.8(j=-2,-1,0,1,2)$ with a center positions at $\Omega=-3.3,-1.7,0,1.7,3.3$ respectively. The spectral filter is applied every $Z=10$ units. The plot of the spectrum and time-evolution is shown one unit in $Z$ after filtering. The figure clearly shows the evolution settles to the desired mode-locked solution. The temporal evolution of the five channels are displayed in Figs. 5(b)-(f). Note that all channels, despite differing group-velocities, are clearly locked into a bound-state which moves at the average group-velocity of the five channels. In this case, symmetry about zero-frequency gives the average group-velocity to be zero. Figure 5(a) further demonstrates 


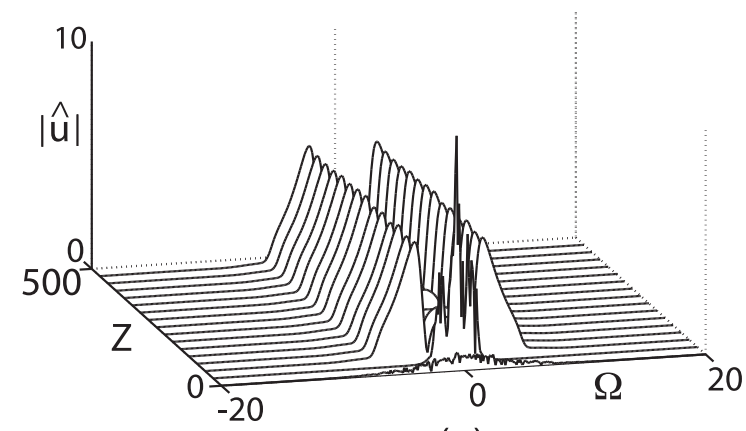

(a)

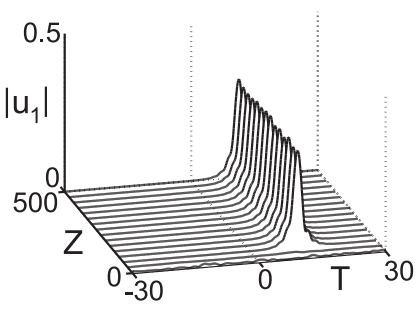

(b)

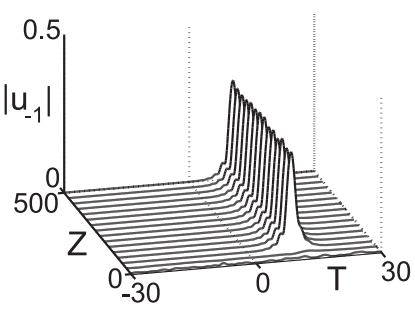

(c)

Figure 4. Illustration of the mode-locking dynamics in the normal dispersion regime with $g_{0}=1.8$. The normalized gain bandwidth is characterized by $\tau=0.01$ so that the FWHM $\Omega_{g}=14.1$. The filter widths are $\Omega_{ \pm 1}=4$ with a center position at $\Omega= \pm 4$ respectively. The spectral filter is applied every $Z=15$ units. The plot of the spectrum (a) and time-evolution (b)-(c) is shown 0.5 units in $Z$ after filtering.

the five-frequency operation. As with the dual-frequency mode-locking, the mode-locking here is highly robust and produces stable mode-locking for a wide range of parameter space.

Figure 6 illustrates the mode-locking dynamics in the anomalous dispersion regime with $g_{0}=1.8$. The normalized gain bandwidth is characterized by $\tau=0.01$ so that the FWHM $\Omega_{g}=14.1$. The filter widths are $\Omega_{j}=2(j=-2,-1,0,1,2)$ with a center positions at $\Omega=-8.7,-4.7,0,4.7,8.7$ respectively. The spectral filter is applied every $Z=15$ units. The plot of the spectrum and time-evolution is shown 0.3 units in $Z$ after filtering. Again the evolution settles to the desired steady bound-state solution shown in Fig. 6(b)-(f). Again, the mode-locking in the anomalous regime is much less robust than in the normal regime. Further, for normal mode-locking the energy is distributed equally between channels while for anomalous the energy follows the gain bandwidth.

To make further connection with experiment, typical values of the mode-locked pulses can be estimated from the simulations presented. For the anomalous regime, if a 30 nanometer gain bandwidth is assumed, then the filter windows assumed in the simulations are approximately 7 nanometers in width. Since the mode-locked pulses are nearly transform limited, this results in mode-locked pulses with a temporal duration on the order of a picosecond. The cavity chromatic dispersion is critical in determining this exact value. In the normal dispersion case, we can turn directly to recent experiments by Wise and co-workers ${ }^{21-23}$ in which sup-picosecond pulses were generated in what is effectively the single-frequency operation of the laser proposed here. As stated earlier, their filter notches were easily tunable in the range from 4-8 nanometers. Thus for multi-frequency operation, the individual channels are expected to have approximately the same pulsewidth and energy as demonstrated in experiments. ${ }^{21-23}$

\section{CONCLUSIONS}

In this paper, it has been shown that the addition of a spectral filter in a laser cavity allows for robust and stable multi- frequency mode-locking in both normal and anomalous dispersion cavities. Indeed, stable and bound-state 


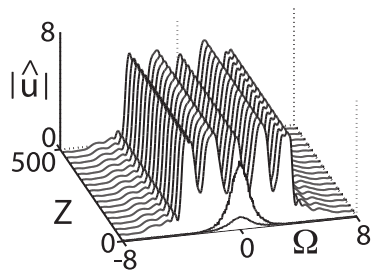

(a)

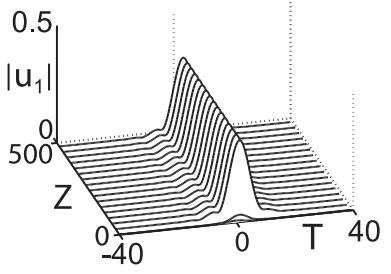

(c)

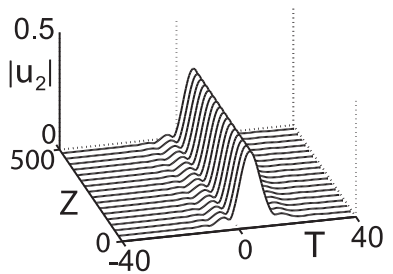

(e)

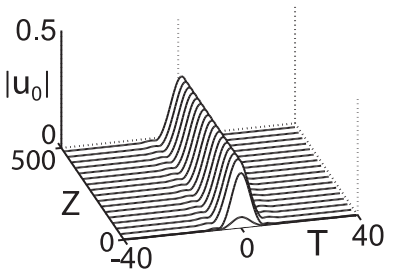

(b)

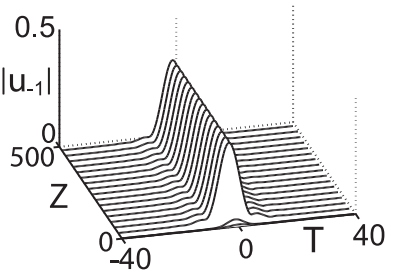

(d)

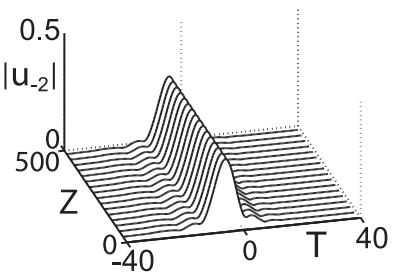

(f)

Figure 5. Illustration of the mode-locking dynamics in the normal dispersion regime with $g_{0}=4.3$. The normalized gain bandwidth is characterized by $\tau=0.1$ so that the FWHM $\Omega_{g}=4.4$. The filter widths are $\Omega_{j}=1(j=-2,-1,0,1,2)$ with a center positions at $\Omega=-3.3,-1.7,0,1.7,3.3$ respectively. The spectral filter is applied every $Z=10$ units. The plot of the spectrum (a) and time-evolution (b)-(f) is shown one unit in $Z$ after filtering.

mode-locking at multiple frequencies can be supported. A theoretical framework for characterizing the modelocking dynamics and stability at the $N$ simultaneous frequencies is developed. Stability is characterized by considering a theoretical model which averages in the effect of the spectral filtering. In doing so, the linear stability can be explicitly calculated in a semi-analytic fashion. This gives significant insight towards understanding both the operation and stability regimes of multi-frequency mode-locked pulsed lasers and recently observed dualfrequency mode-locking experiments. ${ }^{30-33}$

The filter, which is assumed to be a simple notch-type filter with a given bandwidth, acts as a selection mechanism for the multiple frequencies. Of critical importance for mode-locking operation is the cavity length, period of filter application, and output coupler distance from filter. In the normal dispersion regime, stable and robust multi-frequency mode-locking is achieved for a large parameter space. In the anomalous dispersion regime, stable multi-frequency mode-locking is also achieved. However operation is restricted to a smaller parameter space and stable operation is critically dependent upon balancing the key parameters of the cavity length, filter position, and the spectral profile of the filter itself. In particular, it is necessary to have a small filter to output coupler distance compared to the cavity round trip.

Thus the mode-locked bound states formed in the mode-locking process provide an ideal technological basis for multi-frequency photonic applications. Interestingly enough, the mode-locking process counteracts the effects of group- velocity walk-off between neighboring frequency channels, giving rise to a bound-state which propagates at the average group-velocity of the $N$ channels. Given the growing importance of WDM/OTDM technologies, the $N$-channel mode-locking model provides a promising source for stable multiple frequency generation and warrants further exploration. 


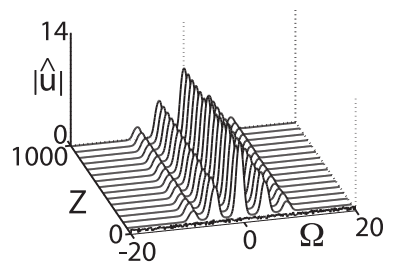

(a)

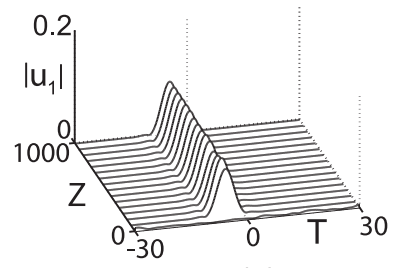

(c)

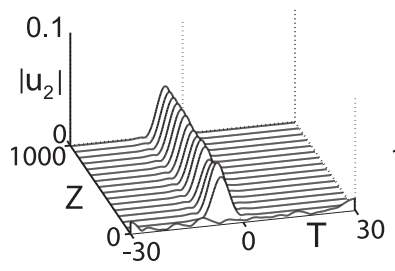

(e)

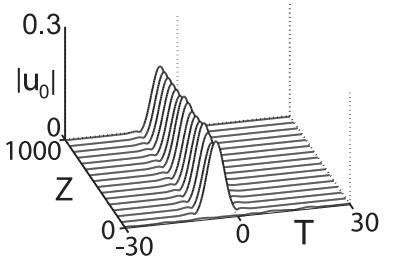

(b)

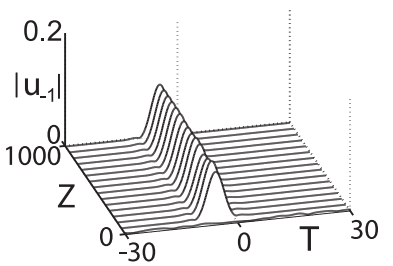

(d)

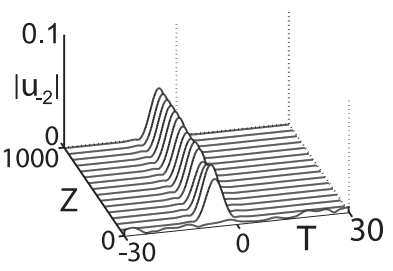

(f)

Figure 6. Illustration of the mode-locking dynamics in the anomalous dispersion regime with $g_{0}=1.8$. The normalized gain bandwidth is characterized by $\tau=0.01$ so that the FWHM $\Omega_{g}=14$. The filter widths are $\Omega_{j}=0.8(j=-2,-1,0,1,2)$ with a center positions at $\Omega=-8.7,-4.7,0,4.7,8.7$ respectively. The spectral filter is applied every $Z=15$ units. The plot of the spectrum (a) and time-evolution (b)-(f) is shown 0.3 units in $Z$ after filtering.

\section{ACKNOWLEDGMENTS}

J. N. Kutz would like to acknowledge support from the National Science Foundation (DMS-0604700).

\section{REFERENCES}

1. G.P. Agrawal, Nonlinear Fiber Optics, Academic Press, New York, 1989.

2. Z. Ahned, N. Onodera Electron Lett. 32, 455 (1996): Z. Li, C. Lou, Y. Gao, K. Chan, Opt. Comm. 185, 381 (2000): C. Wu, N. Dutta, IEEE J. Quant. Elec. 36, 145 (2000)

3. I. N. Duling III and M. L. Dennis, Compact sources of ultrashort pulses. Cambridge, U.K.: Cambridge University Press, 1995.

4. I. N. Duling III, "Subpicosecond all-fiber erbium laser," Electron. Lett., vol. 27, pp. 544-545, 1991.

5. D. J. Richardson, R. I. Laming, D. N. Payne, V. J. Matsas, and M. W. Phillips, "Self-starting, passively modelocked erbium fiber laser based on the amplifying Sagnac switch," Electron. Lett., vol. 27, pp. 542-544, 1991.

6. M. L. Dennis and I. N. Duling III, "High repetition rate figure eight laser with extracavity feedback," Electron. Lett., vol. 28, pp. 2226-2228, 1992.

7. J. W. Haus, G. Shaulov, E. A. Kuzin, and J. Sanchez-Mondragon, "Vector soliton fiber lasers," Opt. Lett., vol. 24, pp. 376-378, 1999.

8. K. Tamura, H. A. Haus, and E. P. Ippen, "Self-starting additive pulse modelocked erbium fiber ring laser," Electro. Lett., vol. 38, pp. 2226-2228, 1992.

9. H. A. Haus, E. P. Ippen, and K. Tamura, "Additive-pulse modelocking in fiber lasers," IEEE J. Quantum. Electron., vol. 30, pp. 200-208, 1994. 
10. M. E. Fermann, M. J. Andrejco, Y. Silverberg, and M. L. Stock, "Passive modelocking by using nonlinear polarization evolution in a polarizing-maintaining erbium-doped fiber laser," Opt. Lett., vol. 29, pp. 447-449, 1993.

11. K. M. Spaulding, D. H. Yong, A. D. Kim, and J. N. Kutz, "Nonlinear dynamics of mode-locking optical fiber ring lasers," J. Opt. Soc. Amer. B, vol. 19, pp. 1045-1054, 2002.

12. F. X. Kartner and U. Keller, "Stabilization of solitonlike pulses with a slow saturable absorber," Opt. Lett., vol. 20, pp. 16-18, 1995.

13. J. N. Kutz, B. C. Collings, K. Bergman, S. Tsuda, S. Cundiff, W. H. Knox, P. Holmes, and M. Weinstein, "Mode-locking pulse dynamics in a fiber laser with a saturable Bragg reflector," J. Opt. Soc. Amer. B, vol. 14, pp. 2681-2690, 1997.

14. S. Tsuda, W. H. Knox, E. A. DeSouza, W. J. Jan, and J. E. Cunningham, "Low-loss intracavity AlAs/AlGaAs saturable Bragg reflector for femtosecond modelocking in solid-state lasers," Opt. Lett., vol. 20, pp. 1406-1408, 1995 .

15. F. Krausz, M. E. Fermann, T. Brabec, P. F. Curley, M. Hofer, M. H. Ober, C. Speilmann, E. Wintner, and A. J. Schmit, "Femtosecond solid-state lasers," IEEE J. Quantum Electron., vol. 28, pp. 2097-2122, 1992.

16. H. A. Haus, J. G. Fujimoto, and E. P. Ippen, "Structures for additive pulse modelocking," J. Opt. Soc. Amer. B, vol. 8, pp. 2068-2076, 1991.

17. J. Proctor and J. N. Kutz, "Passive mode-locking by use of waveguide arrays," Opt. Lett., vol. 30, pp. 20132015, 2005.

18. J. Proctor and J. N. Kutz, " Nonlinear mode-coupling for passive mode-locking: application of waveguide arrays, dual-core fibers, and/or fiber arrays," Opt. Express, vol. 13, pp. 8933-8950, 2005.

19. J. Proctor, J.N. Kutz, "Averaged models for passive mode-locking using nonlinear mode-coupling," J. Math and Comp. in Sim. 74:333-342, 2007

20. J. N. Kutz, B. Sandstede, "Theory of passive harmonic mode-locking using wave-guide arrays," Opt. Express, vol. 16, pp. 636-650, 2008.

21. A. Chong, J. Buckley, W. Renninger, and F. Wise, "All-normal-dispersion femtosecond fiber laser," Opt. Express, vol. 14, pp. 10095-10100, 2006.

22. A. Chong, W. H. Renninger, and F. W. Wise, "All-normal-dispersion femtosecond fiber laser with pulse energy above 20nJ," Opt. Lett., vol. 32, pp. 2408-2410, 2007.

23. B.G. Bale, J.N. Kutz, A. Chong, W.H. Renninger, and F.W. Wise, "Spectral Filtering for Ultra-fast Modelocking in the Normal Dispersive Regime," Opt. Lett. vol. 33, pp. 941-943, 2008.

24. A. Hasegawa and Y. Kodama, "Guiding-center soliton in optical fibers," Opt. Lett., vol. 15, pp. 1443-1445, 1990.

25. E. Farnum, L. Butson, and J. N. Kutz, "Theory and simulation of dual-frequency mode-locked lasers", $J$. Opt. Soc. Am. B, vol. 23, pp. 257-264, 2006.

26. N. R. Pereira, L. Stenflo, "Nonlinear Schrödinger equation including growth and damping," Phys. Fluids, vol. 20, pp. 1733-1744, 1977.

27. P. G. Drazin, Nonlinear Systems. Cambridge, U.K.: Cambridge University Press, 1992.

28. B. Deconinck, J. N. Kutz, "Computing spectra of linear operators using Hill's method", J. Comp. Physics, vol. 219, pp. 296-321, 2006.

29. H. Goldstein, Classical Mechanics. Addison-Wesley, 1980.

30. Y. Shiquan, L. Zhaohui, Y. Shuzhong, D. Xiaoyyi, K. Guiyun, and Z. Qida, "Dual-wavelength actively modelocked erbium dobed fiber laser using FBGs," Proceedings of SPIE Vol. 4974, $43-49$ (SPIE, Bellingham, WA 2003).

31. H. Dong, G. Zhu, Q. Wang, and N. K. Dutta, "Simultaneous mode locked operation of a fiber laser at two wavelengths," Proceedings of SPIE Vol. 5349, 117-121 (SPIE, Bellingham, WA 2004).

32. Z. Ahned and N. Onodera, "High repetition rate optical pulse generation by frequency multiplication in actively mode-locked fiber ring lasers," Elec. Lett. 32, 455-455 (1996).

33. C. Wu and N. K. Dutta, "High repetition-rate optical pulse generation using a rational harmonic mode-locked fiber laser," IEEE J. Quant. Elec. 36, 145-150 (2000). 